\title{
WEAK TYPE $(1,1)$ ESTIMATES FOR HEAT KERNEL MAXIMAL FUNCTIONS ON LIE GROUPS
}

\author{
MICHAEL COWLING, GARTH GAUDRY, SAVERIO GIULINI AND \\ GIANCARLO MAUCERI
}

\begin{abstract}
For a Lie group $G$ with left-invariant Haar measure and associated Lebesgue spaces $L^{p}(G)$, we consider the heat kernels $\left\{p_{t}\right\}_{t>0}$ arising from a right-invariant Laplacian $\Delta$ on $G$ : that is, $u(t, \cdot)=p_{t} * f$ solves the heat equation $(\partial / \partial t-\Delta) u=0$ with initial condition $u(0, \cdot)=f(\cdot)$. We establish weak-type $(1,1)$ estimates for the maximal operator $\mathscr{M}\left(\mathscr{M} f=\sup _{t>0}\left|p_{t} * f\right|\right)$ and for related Hardy-Littlewood maximal operators in a variety of contexts, namely for groups of polynomial growth and for a number of classes of Iwasawa $A N$ groups. We also study the "local" maximal operator $\mathscr{M}_{0}\left(\mathscr{K}_{0} f=\right.$ $\left.\sup _{0<t<1}\left|p_{t} * f\right|\right)$ and related Hardy-Littlewood operators for all Lie groups.
\end{abstract}

\section{INTRODUCTION}

Let $G$ be a connected Lie group, with fixed left-invariant Haar measure $d x$, associated Lebesgue spaces $L^{p}(G)$, and modular function $\delta$, so that

$$
\int_{G} f(x y) d x=\delta(y)^{-1} \int_{G} f(x) d x .
$$

As usual, we identify the Lie algebra $\mathfrak{g}$ of $G$ with the tangent space at the origin, and denote by exp the exponential mapping. Let $\left\{X_{1}, \ldots, X_{d}\right\}$ be a basis for $\mathfrak{g}$ and take $k$ in $[1, d]$ such that $\left\{X_{1}, \ldots, X_{k}\right\}$ generates $\mathfrak{g}$ as a Lie algebra. Define the corresponding sub-Laplacean $\Delta$ to be the right-invariant differential operator on $C_{c}^{\infty}(G)$ given by the formula

$$
\Delta f(x)=\left.\sum_{j=1}^{k}(d / d t)^{2} f\left(\exp \left(t X_{j}\right) x\right)\right|_{t=0} \quad \forall x \in G ;
$$

$\Delta$ may also be considered as a densely-defined operator on $L^{p}(G)$.

We may associate to $\Delta$ a family of heat kernels $\left\{p_{t}\right\}_{t>0}$ such that

(i) $p_{t} \in C^{\infty}(G)$ for all $t$ in $\mathbf{R}^{+}$;

(ii) $\delta(x) p_{t}(x)=p_{t}\left(x^{-1}\right)$ and $p_{t} \geq 0$ for all $t$ in $\mathbf{R}^{+}$;

Received by the editors February 24, 1989.

1980 Mathematics Subject Classification (1985 Revision). Primary 22E30; Secondary 42B25.

Research supported by the Australian Research Grants Scheme, the Italian Consiglio Nazionale delle Ricerche, and the Italian Ministero della Pubblica Istruzione. 
(iii) $\left\|p_{t}\right\|_{1}=1$ for all $t$ in $\mathbf{R}^{+}$;

(iv) $\partial / \partial t\left(p_{t} * f\right)=\Delta\left(p_{t} * f\right)$ for all $t$ in $\mathbf{R}^{+}$and every $f$ in $L^{p}(G), 1 \leq$ $p \leq \infty$;

(v) $\lim _{t \rightarrow 0+} p_{t} * f=f$ for every $f$ in $L^{p}(G)$, where the limit is in the $L^{p}$-norm if $1 \leq p<+\infty$, and in the weak-star topology if $p=\infty$.

A relaxed account of the theory in the case where $\Delta$ is a Laplacean (i.e. $k=d$ ) can be found in A. Hulanicki [7]. In the general case, some modifications must be made to take into account the fact that $\Delta$ is subelliptic rather than elliptic. However, using the theory developed by L. Hörmander [6], this is straightforward.

In this paper, we consider the heat semigroup $\left\{P_{t}\right\}_{t>0}$, where $P_{t}=p_{t} *$. In [11], E. M. Stein showed that the maximal operator $\mathscr{M}$ associated to the heat kernels

$$
\mathscr{M} f=\sup _{t>0}\left|p_{t} * f\right| \quad \forall f \in L^{p}(G)
$$

is bounded on $L^{p}(G)$ provided $1<p \leq \infty$. We show here that, if $\mathscr{M}_{0}$ is the "local" heat maximal operator

$$
\mathscr{M}_{0} f=\sup _{0<t<1}\left|p_{t} * f\right| \quad \forall f \in L^{p}(G)
$$

then $\mathscr{M}_{0}$ is of weak type $(1,1)$ for all connected Lie groups $G$, and that $\mathscr{M}$ is of weak type $(1,1)$ for many Lie groups, namely groups of polynomial growth and certain Iwasawa $A N$ groups. We also show that, in these cases, the heat kernels resemble the heat kernels on $\mathbf{R}^{n}\left((4 \pi t)^{-n / 2} e^{-|x|^{2} / 4 t}\right)$ sufficiently closely that we can prove weak $(1,1)$ estimates for the Hardy-Littlewood maximal operator obtained by averaging over "balls" in $G$.

The techniques we use to study maximal functions are simple, and we illustrate them in the setting where $G=\mathbf{R}^{n}$ and $\Delta$ is the standard Laplacean. Since $\left\|p_{t} * f\right\|_{p} \leq\|f\|_{p}$ for all $f$ in $L^{p}\left(\mathbf{R}^{n}\right)$ and for all $p$ in $[1, \infty]$, the Hopf-Dunford-Schwartz maximal ergodic theorem [2, p. 690] implies that, if we define $a_{t}\left(t\right.$ in $\left.\mathbf{R}^{+}\right)$and $\mathscr{E}$ by the rules

$$
a_{t}=\frac{1}{t} \int_{t}^{2 t} p_{s} d s
$$

and

$$
\mathscr{E} f=\sup _{t>0}\left|a_{t} * f\right| \quad \forall f \in L^{p}\left(\mathbf{R}^{n}\right)
$$

then $\mathscr{E}$ is of weak type $(1,1)$. Now, for any $x$ in $\mathbf{R}^{n}$,

$$
\begin{aligned}
a_{t}(x) & =\frac{1}{t} \int_{t}^{2 t}(4 \pi)^{-n / 2} s^{-n / 2} e^{-|x|^{2} / 4 s} d s \\
& \geq(4 \pi)^{-n / 2} \frac{(2 t)^{-n / 2}}{t} \int_{t}^{2 t} e^{-|x|^{2} / 4 s} d s \\
& \geq(4 \pi)^{-n / 2}(2 t)^{-n / 2} e^{-|x|^{2} / 4 t}=2^{-n / 2} p_{t}(x) .
\end{aligned}
$$

It follows that $\mathscr{M}$ is of weak type $(1,1)$. 
Finally,

$$
\begin{aligned}
p_{t}(x) & =(4 \pi)^{-n / 2} t^{-n / 2} e^{-|x|^{2} / 4 t} \geq(4 \pi)^{-n / 2} t^{-n / 2} e^{-1} \chi_{\left\{u:|u|^{2} \leq 4 t\right\}}(x) \\
& \geq 2(n e \Gamma(n / 2))^{-1}\left|\left\{u \in \mathbf{R}^{n}:|u|^{2} \leq 4 t\right\}\right|^{-1} \chi_{\left\{u:|u|^{2} \leq 4 t\right\}}(x),
\end{aligned}
$$

and so the weak type $(1,1)$ boundedness of the Hardy-Littlewood maximal operator, defined by taking averages over Euclidean balls, follows from the boundedness of the heat maximal operator $\mathscr{M}$.

In order to be able to estimate the heat kernels $p_{t}$ by (a multiple of) the ergodic kernels $a_{t}$ as above, we need a reasonably explicit formula for $p_{t}$. The general theory of the heat equation provides enough information to allow us to treat the local maximal function $\mathscr{M}_{0}$ on all Lie groups. On the other hand, the estimates which are presently available (see e.g. S. Kusuoko and D. Stroock [9] or N. Varopoulos [12]) allow us to establish the weak $(1,1)$ boundedness of the operator $\mathscr{M}$ when $G$ is of polynomial growth.

For the Iwasawa $A N$ groups, we study a canonical Laplacean, viz one generated by an orthonormal basis of $\mathfrak{g}$ and a basis of root vectors in $\mathfrak{n}$. We establish a relationship between the heat kernels on $A N$ and the heat kernels associated with the Laplace-Beltrami operator $\mathscr{L}$ on the symmetric space $G / K$. (The group $G$ is assumed to have the Iwasawa decomposition $G=A N K$; then $G / K \cong A N$, as smooth manifolds.) Detailed information is available about the heat kernels on $G / K$ for large classes of symmetric spaces; e.g. N. Lohoué and Th. Rychener [10] and J.-P. Anker and N. Lohoue [1]. Using their results and the relationship between the heat kernels on $A N$ (for $\Delta$ ) and on $G / K$ (for $\mathscr{L}$ ) we prove that $\mathscr{M}$ is of weak type $(1,1)$ in the cases when $G / K$ is of rank $1, G$ is complex, or $\mathfrak{g}$ is a normal real form.

At an early stage of this work, we had established a special case of the relationship between heat kernels on $A N$ and those on $G / K$ (Theorem 5.2). We thank M. Flensted-Jensen for pointing out the result is general. The proofs given in 5.1-5.2 are due essentially to him.

\section{Preliminaries}

Suppose that $G$ is a connected Lie group and $X_{1}, \ldots, X_{k}$ generate $\mathfrak{g}$. Denote also by $X_{1}, \ldots, X_{k}$ the right-invariant vector fields agreeing with the respective elements of the tangent space at $e$; so

$$
X_{j} f(x)=\left.\frac{d}{d t} f\left(\exp \left(t X_{j}\right) x\right)\right|_{t=0} .
$$

Define

$$
\Delta=\sum_{j=1}^{k} X_{j}^{2} .
$$

The right-invariant operator $\Delta$ is selfadjoint and negative. 
Associate a right-invariant distance $d$ on $G$ to $\left\{X_{1}, \ldots, X_{k}\right\}$ as follows. Let $P$ be the set of all piecewise continuously differentiable mappings $\gamma:[0,1] \rightarrow G$ such that

$$
\dot{\gamma}(t)=\sum_{j=1}^{k} a_{k}(t) X_{j}(\gamma(t)) \quad \text { in } T_{\gamma(t)}(G),
$$

for all but finitely many $t$. Define the length of $\gamma$, denoted $|\gamma|$, by the rule

$$
|\gamma|=\int_{0}^{1}\left(\sum_{j=1}^{k}\left|a_{j}(t)\right|^{2}\right)^{1 / 2} d t .
$$

Then define $d$ by the formula

$$
d(x, y)=\inf \{|\gamma|: \gamma \in P, \gamma(0)=x, \gamma(1)=y\} \quad \forall x, y \in G .
$$

Denote by $B(x, r)$ the ball, centred at $x$, of radius $r$; set $|x|=d(x, e)$.

It can be shown that $d$ is a metric on $G$ which induces the usual topology, and which comes from a Riemannian metric when $X_{1}, \ldots, X_{k}$ span $\mathfrak{g}$. A convenient reference for these facts is [12].

Consider now the heat diffusion boundary value problem in $\mathbf{R}^{+} \times G$ :

$$
\left\{\begin{array}{l}
(\partial / \partial t-\Delta) u=0 \\
u(t, \cdot) \rightarrow f(\cdot) \quad \text { as } t \rightarrow 0+.
\end{array}\right.
$$

With the appropriate modifications to take care of the fact that $\left\{X_{1}, \ldots, X_{k}\right\}$ is not necessarily a basis of $\mathfrak{g}$, Theorem 3.4 of [7] shows that there is a unique semigroup $\left\{P_{t}\right\}_{t>0}$ of operators and a corresponding convolution semigroup $\left\{p_{t}\right\}_{t>0}$ of kernels, with properties like those of the Gaussian kernels in the Euclidean setting, such that, if $f \in L^{p}(G)$ and $u(t, \cdot)=p_{t} * f(\cdot)$, then $u$ is the unique solution to (2), it being understood that the boundary value is taken in the weak-star topology if $p=\infty$.

It is important for us to have precise information about the kernels of the convolution semigroup $\left\{p_{t}\right\}_{t>0}$, but this is difficult for arbitrary Lie groups $G$, principally because different classes of Lie groups may behave in very different ways at infinity. However, all Lie groups are locally Euclidean, so that useful general local estimates can be proved. To state these, we denote by $\beta$ that positive number for which $r^{-\beta}|B(e, r)|$ is bounded and bounded away from 0 for all $r$ in $(0,1]$. See [12].

Theorem 2.1. Fix $T$ in $\mathbf{R}^{-}$. Then there are nonnegative constants $C, C^{\prime}$, and $C^{\prime \prime}$ such that

(a) whenever $0<t \leq T$ and $x \in B(e, 1)$,

$$
\frac{1}{C} t^{-\beta / 2} \exp \left(-|x|^{2} / C^{\prime} t\right) \leq p_{t}(x) \leq C t^{-\beta / 2} \exp \left(-|x|^{2} / C^{\prime \prime} t\right) ;
$$

(b) whenever $0 \leq t \leq T$ and $x \in G \backslash B(e, 1)$,

$$
p_{t}(x) \leq h(x)
$$

where $h(x)=O\left(e^{-\lambda|x|}\right)$ for all $\lambda$ in $\mathbf{R}^{+}$. In particular, $h \in L^{1}(G)$. 
Proof. These inequalities follow from the estimates of Kusuoka and Stroock [9] or Varopoulos [12, §4].

\section{LOCAL MAXIMAL OPERATORS}

Let the notation be as in $\S 2$.

Definition 3.1. For each measurable function $f$ on $G$ for which the following formulae make sense, let

$$
\mathscr{M}_{0} f=\sup _{0<t<T}\left|p_{t} * f\right|
$$

and

$$
\mathscr{H}_{0} f=\sup _{0<t<T}|B(e, t)|^{-1}\left|\chi_{B(e, t)} * f\right| .
$$

The operators $\mathscr{M}_{0}$ and $\mathscr{H}_{0}$ are called the local heat maximal operator and the local Hardy-Littlewood maximal operator respectively.

Observe that the mapping properties of the operators $\mathscr{M}_{0}$ and $\mathscr{H}_{0}$ on the $L^{p}$-spaces are independent of the choice of $T$. For $\mathscr{H}_{0}$, this is because, if $0<a<b$, then

$$
\sup _{a<t<b}|B(e, t)|^{-1}\left|\chi_{B(e, t)} * f\right| \leq|B(e, a)|^{-1} \chi_{B(e, b)} *|f|,
$$

and the function $|B(e, a)|^{-1} \chi_{B(e, b)}$ is integrable. Similar reasoning applies to $\mathscr{M}_{0}$.

Theorem 3.2. The operators $\mathscr{M}_{0}$ and $\mathscr{H}_{0}$ are of weak type $(1,1)$.

Proof. (i) Consider first the operator $\mathscr{M}_{0}$. By virtue of part (b) of Theorem 2.1,

$$
p_{t}(x) \leq h(x)
$$

whenever $x \in G \backslash B(e, 1)$ and $0<t \leq T$, where $h \in L^{1}(G)$.

On the other hand, if $x \in B(e, 1)$ and $t<T / 2$, from part (a) of Theorem 2.1 we see that

$$
a_{t}(x)=\frac{1}{t} \int_{t}^{2 t} p_{s}(x) d x \geq \frac{1}{C}(2 t)^{-\beta / 2} \exp \left(-|x|^{2} / C^{\prime} t\right) \geq D p_{\lambda t}(x)
$$

for appropriate positive constants $D$ and $\lambda$. From (1) and (2), there is a positive constant $E$ such that

$$
p_{t}(x) \leq E\left[h(x)+a_{\lambda^{-1} t}(x)\right] \quad \forall x \in G,
$$

when $t \leq T / 2$. Now by the Hopf-Dunford-Schwartz theorem, the maximal operator $\mathscr{E}$

$$
\mathscr{E}: f \mapsto \sup _{0<t \leq T / 2} a_{\lambda^{-1} t} *|f|
$$

is of weak type $(1,1)$. The operator $f \mapsto h * f$ is of strong type $(1,1)$. Hence $\mathscr{M}_{0}$ is of weak type $(1,1)$. 
(ii) To treat the operator $\mathscr{H}_{0}$, we remark that, from part (a) of Theorem 2.1, there are constants $D$ and $D^{\prime}$ such that, if $0<t \leq T$ and $x \in G$,

$$
t^{-\beta / 2} \chi_{B\left(e, t^{1 / 2}\right)}(x) \leq D t^{-\beta / 2} \exp \left(-|x|^{2} / C^{\prime} t\right) \leq D^{\prime} p_{t}(x) .
$$

The weak $(1,1)$ boundedness of $\mathscr{H}_{0}$ follows from that of $\mathscr{M}_{0}$ and the earlier remarks about the freedom in the choice of $T$.

\section{GROUPS OF POLYNOMIAL GROWTH}

Let $G$ be a group of polynomial growth, i.e. such that for any relatively compact neighbourhood $U$ of $e$ in $G,\left|U^{n}\right|$ grows polynomially in $n$. (We recall that all Euclidean motion groups and all nilpotent groups have this property.) Then there exists $\gamma$ in $[0,+\infty)$, such that $n^{-\gamma}\left|U^{n}\right|$ is bounded above and away from 0 for all such neighbourhoods $U$ [8]. In particular, in our general framework, there are positive constants $C$ and $C^{\prime}$ such that

$$
C \leq r^{-\gamma}|B(e, r)| \leq C^{\prime} \quad \forall r \in[1,+\infty),
$$

and from [9] it follows that there are positive constants $A, B, C_{1}, C_{2}$, such that

$$
C_{1} t^{-\gamma / 2} \exp \left(-|x|^{2} / A t\right) \leq p_{t}(x) \leq C_{2} t^{-\gamma / 2} \exp \left(-|x|^{2} / B t\right)
$$

whenever $t \geq 1$ and $x \in G$. See also [12].

Theorem 4.1. If $G$ is of polynomial growth, the maximal operators $\mathscr{M}$ and $\mathscr{H}$ are of weak type $(1,1)$.

Proof. The boundedness of $\mathscr{M}_{0}$ and $\mathscr{H}_{0}$ is taken care of in Theorem 3.2. The proof of the boundedness of $\mathscr{M}_{\infty}$,

$$
\mathscr{M}_{\infty}: f \mapsto \sup _{t \geq 1}\left|p_{t} * f\right|
$$

follows the same lines as that of $\mathscr{M}_{0}$, once (1) is available.

The weak $(1,1)$ boundedness of $\mathscr{H}$ is, as usual, a corollary of the result for $\mathscr{M}$.

\section{IWASAWA $A N$ GROUPS}

Let $G$ denote a connected, noncompact semisimple Lie group and $\mathfrak{g}$ its Lie algebra. Denote by $\theta$ a Cartan involution of $\mathfrak{g}$, and write

$$
\mathfrak{g}=\mathfrak{k} \oplus \mathfrak{p}
$$

for the associated Cartan decomposition. Fix a maximal abelian subspace $\mathfrak{a}$ of $\mathfrak{p}$; this determines a root space decomposition

$$
\mathfrak{g}=\mathfrak{g}^{0} \oplus \bigoplus_{\alpha \in \Sigma} \mathfrak{g}^{\alpha},
$$


$\Sigma$ denoting the set of roots of the pair $(\mathfrak{g}, \mathfrak{a})$. Corresponding to a choice of ordering of the roots, we have an Iwasawa decomposition

$$
\mathfrak{g}=\mathfrak{k} \oplus \mathfrak{a} \oplus \mathfrak{n}=\mathfrak{k} \oplus \mathfrak{a} \oplus \bigoplus_{\alpha \in \Sigma^{+}} \mathfrak{g}^{\alpha}
$$

further

$$
\mathfrak{g}=\mathfrak{m} \oplus \mathfrak{a} \oplus \mathfrak{n} \oplus \theta \mathfrak{n},
$$

$\mathfrak{m}$ denoting the centraliser of $\mathfrak{a}$ in $\mathfrak{k}$.

Write $G=A N K$ for the Iwasawa decomposition of $G$ corresponding to (1). The solvable group $A N$ is identifiable, as a manifold, with the symmetric space $G / K$. Smooth functions on $A N$ will be identified with smooth right $K$-invariant functions on $G$.

The Laplacean on $A N$. A distinguished Laplacean on $A N$ can be constructed as follows. Choose an orthonormal basis in $\mathfrak{a}$, say $\left\{H_{1}, \ldots, H_{l}\right\}$ and let

$$
\omega_{\mathfrak{a}}=\sum_{j=1}^{l} H_{j}^{2} .
$$

Denote by $P_{+}$the set of roots $\alpha$ of the complexification $\mathfrak{g}_{\mathbf{C}}$ whose restriction $\widehat{\alpha}$ to $\mathfrak{a}$ is nonzero. For each $\alpha$ in $P_{+}$, choose $X_{\alpha}$ in $\mathfrak{g}^{\hat{\alpha}}$ such that $X_{-\alpha}=\theta X_{\alpha}$ and

$$
B\left(X_{\alpha}, \theta X_{\beta}\right)=-2 \delta_{\alpha \beta} \quad \forall \alpha, \beta \in P_{+},
$$

$B$ denoting the Killing form on $\mathfrak{g}$.

Define the Laplacean $\Delta$ by setting

$$
\Delta=\omega_{\mathfrak{a}}+\sum_{\alpha \in P_{+}} X_{\alpha}^{2} .
$$

Proposition 5.1. (i) The Casimir operator on $G$ is given by the formula

$$
\Omega=\Omega_{\mathfrak{a}}-\Omega_{\mathfrak{m}}-\frac{1}{2} \sum_{\alpha \in P_{+}}\left(X_{\alpha} X_{-\alpha}+X_{-\alpha} X_{\alpha}\right),
$$

where $\Omega_{\mathfrak{m}}$ is the sum of squares of the elements of a basis, orthonormal with respect to $-B$, in $\mathfrak{m}$.

(ii) The Laplace-Beltrami operator $\mathscr{L}$ for $G / K$ acting on $C^{\infty}(G / K)$ can be identified with the operator $\Omega$ on $C^{\infty}(G / K)$.

Proof. The first result follows from the definitions. Because $\Omega$ is rightinvariant, $\Omega$ maps $C^{\infty}(G / K)$ to itself, and because $\Omega$ comes from an $\operatorname{Ad}(G)$ invariant expression in the enveloping algebra of $\mathfrak{g}$, it is also left-invariant, hence is identifiable with the Laplace-Beltrami operator. See also [4, p. 331].

The group $A N$ has left Haar measure $d a d n$ where $d a$ and $d n$ are the biinvariant measures on $A$ and $N$ respectively. The modular function $\delta$ on $A N$ is given by the formula

$$
\delta(a n)=e^{-2 \rho(\log a)} \quad \forall a n \in A N,
$$


$\rho$ denoting one-half of the sum of the positive (restricted) roots, counted with multiplicity. As remarked above, we shall also regard $\delta$ as a $K$-right-invariant function on $G$.

Theorem 5.2. (i) Suppose $f \in C^{\infty}(K \backslash G)$. Then

$$
\delta^{1 / 2} \Delta \delta^{-1 / 2} f=\Omega f+\langle\rho, \rho\rangle f,
$$

where $\langle\cdot, \cdot\rangle$ denotes the inner product dual to the Killing form on $\mathfrak{a}^{*}$.

(ii) If $f \in C^{\infty}(K \backslash G / K)$, then

$$
\delta^{1 / 2} \Delta \delta^{-1 / 2} f=\mathscr{L} f+\langle\rho, \rho\rangle f .
$$

Proof. (i) Since $f$ is invariant on the left under $K$,

$$
\Omega f=\Omega_{\mathrm{a}} f-\frac{1}{2} \sum_{\alpha \in P_{+}}\left(X_{\alpha} X_{-\alpha}+X_{-\alpha} X_{\alpha}\right) f .
$$

Since $X_{\alpha}+X_{-\alpha}$ is in $\mathfrak{k}$, we have $\left(X_{\alpha}+X_{-\alpha}\right) f=0$. Moreover, $\left[X_{\alpha}, X_{-\alpha}\right]=$ $-2 H_{\hat{\alpha}}$, and so $X_{-\alpha} X_{\alpha} f-X_{\alpha} X_{-\alpha} f=-2 H_{\hat{\alpha}} f$, since commutators of rightinvariant vector fields correspond to minus the corresponding Lie bracket. Now we see that

$$
\left(X_{\alpha} X_{-\alpha}+X_{-\alpha} X_{\alpha}\right) f=2 X_{\alpha} X_{-\alpha} f-2 H_{\hat{\alpha}} f=-2 X_{\alpha}^{2} f-2 H_{\hat{\alpha}} f .
$$

It follows from (2) that

$$
\begin{aligned}
\Omega f & =\Omega_{\mathfrak{a}} f+\sum_{\alpha \in P_{+}}\left(H_{\hat{\alpha}}+X_{\alpha}^{2}\right) f \\
& =\Omega_{\mathfrak{a}} f+H_{2 \rho} f+\sum_{\alpha \in P_{+}} X_{\alpha}^{2} f=\Delta f+H_{2 \rho} f .
\end{aligned}
$$

Now

$$
\begin{aligned}
\Delta\left(\delta^{-1 / 2} f\right) & =\left(\Omega_{\mathfrak{a}}+\sum_{\alpha \in P_{+}} X_{\alpha}^{2}\right)\left(\delta^{-1 / 2} f\right), \\
& =\Omega_{\mathfrak{a}}\left(\delta^{-1 / 2} f\right)+\delta^{-1 / 2} \sum_{\alpha \in P_{+}} X_{\alpha}^{2} f,
\end{aligned}
$$

since $\delta$ is $N$-invariant. For each $j$,

$$
H_{j}\left(\delta^{-1 / 2} f\right)(\text { ank })=\rho\left(H_{j}\right) \delta^{-1 / 2}(\text { an }) f(\text { ank })+\delta^{-1 / 2}(\text { an }) H_{j} f(\text { ank }) ;
$$

it follows that

$$
H_{j}^{2}\left(\delta^{-1 / 2} f\right)=\rho\left(H_{j}\right)^{2} \delta^{-1 / 2} f+2 \rho\left(H_{j}\right) \delta^{-1 / 2} H_{j} f+\delta^{-1 / 2} H_{j}^{2} f,
$$

and so

$$
\Omega_{\mathfrak{a}}\left(\delta^{-1 / 2} f\right)=\langle\rho, \rho\rangle \delta^{-1 / 2} f+\delta^{-1 / 2} H_{2 \rho} f+\delta^{-1 / 2} \Omega_{\mathfrak{a}} f,
$$

since the $H_{j}$ commute. From (3) and (4) we conclude that

$$
\delta^{1 / 2} \Delta \delta^{-1 / 2} f=\langle\rho, \rho\rangle f+H_{2 \rho} f+\Omega_{\mathfrak{a}} f+\sum_{\alpha \in P_{+}} X_{\alpha}^{2} f=\Omega f+\langle\rho, \rho\rangle f .
$$


(ii) If $f$ is also right- $K$-invariant, then

$$
\Omega f=\mathscr{L} f
$$

by part (ii) of Proposition 5.1.

Heat kernels on AN groups. We shall now exploit the connection between the right-invariant Laplacean $\Delta$ on $A N$ and the Laplace-Beltrami operator $\mathscr{L}$ on $G / K$, given in Theorem 5.2, to give an explicit expression for the heat kernels associniad to $\Delta$.

Let $\left\{q_{t}\right\}_{t>0}$ be the heat kernels on $G / K$ associated with $\mathscr{L}$. The functions $q_{t}$ are regarded as right- $K$-invariant functions on $G$, or by restriction as functions on $A N$.

Theorem 5.3. The family $\left\{p_{t}\right\}_{t>0}$ given by the formula

$$
p_{t}=e^{\langle\rho, \rho\rangle t} \delta^{-1 / 2} q_{t}
$$

is the family of heat kernels for the operator $\Delta$ on $A N$.

Proof. The operator $\Delta$ is the infinitesimal generator of a $C_{0}$ semigroup. Associated with $\Delta$ there is a canonical semigroup of probability measures, say $\left\{p_{t}^{\prime}\right\}_{t>0}$ [7]. We wish to show that $p_{t}=p_{t}^{\prime}$ for all $t$.

Fix $f$ in $C_{c}^{\infty}(A N)$. The abstract Cauchy problem $A C P_{2}$ with boundary data $f$ has a unique solution $u^{\prime}$ [5, Theorem 23.8.1; see also p. 619], viz

$$
u^{\prime}(t, \cdot)=p_{t}^{\prime} * f(\cdot)
$$

Once it is shown that, if

$$
u(t, \cdot)=p_{t} * f(\cdot),
$$

then $u$ is also a solution to the same Cauchy problem, it will follow that, for all $t$ in $\mathbf{R}^{+}$.

$$
p_{t} * f=p_{t}^{\prime} * f
$$

for all $f$ in $C_{c}^{\infty}(A N)$, whence $p_{t}=p_{t}^{\prime}$.

It is shown in [7, Proposition 4.1] that, for every $\alpha$ in $\mathbf{R}^{+}$and $t$ in $(0, R)$,

$$
\int_{A N} p_{t}^{\prime}(x) e^{\alpha|x|} d x<C(\alpha, R),
$$

where $|x|$ denotes the Riemannian distance from $e$, canonically associated with the choice of basis of $\mathfrak{g}$. Let $s$ be negative, and consider the weight function $w_{s}$

$$
w_{s}(x)=e^{s|x|} \quad \forall x \in G
$$


Denote by $P_{t}^{\prime}$ the convolution operator defined by $p_{t}^{\prime}(t>0)$. Then if $f \in$ $C_{c}(A N)$ and $0<t<R$,

$$
\begin{aligned}
\left\|P_{t}^{\prime} f\right\|_{L^{\prime}\left(w_{s}\right)} & =\int_{A N}\left|p_{t}^{\prime} * f(x)\right| w_{s}(x) d x \\
& \leq \int_{A N} \int_{A N}\left|p_{t}^{\prime}(y) f(x)\right| w_{s}(y x) d x d y \\
& \leq \int_{A N} \int_{A N} p_{t}^{\prime}(y) e^{-s|y|} e^{s|x|}|f(x)| d x d y \\
& \leq C(-s, R)\|f\|_{L_{1}\left(w_{s}\right)}
\end{aligned}
$$

Since $P_{t}^{\prime}$ clearly carries $L^{\infty}(A N)$ into $L^{\infty}(A N)$, it follows by interpolation that $\left\{P_{t}^{\prime}\right\}_{t>0}$ can be thought of as a semigroup of operators on $L^{2}\left(w_{s}\right)$. The infinitesimal generator of $\left\{P_{t}^{\prime}\right\}_{t>0}$ is $\Delta$. Moreover, $\left\{P_{t}^{\prime}\right\}$ is a $C_{0}$-semigroup. Consider a fixed $f$ in $C_{c}^{\infty}(A N)$, and the abstract Cauchy problem. $A C P_{2}$ with values in $L^{2}\left(w_{s}\right)$; see Hille-Phillips [5, pp. 619-622]. We shall show in (i)-(iii) that the function $u(t, \cdot)=p_{t} * f(\cdot)$ is a solution to this problem, provided $s$ is sufficiently large negative, whence by Theorem 23.8 .1 of [5], it will follow that $p_{t} * f=p_{t}^{\prime} * f$ for all $t$ in $\mathbf{R}^{+}$.

(i) $u$ satisfies the heat equation on $(0, \infty) \times A N$.

(ii) $u(t, \cdot) \rightarrow f(\cdot)$ in $L^{2}\left(w_{s}\right)$ as $t \rightarrow 0+$.

(iii) $u$ is absolutely continuous with continuous derivative on each compact subinterval of $\mathbf{R}^{+}$provided $s$ is sufficiently large negative. To see this, write, using the inversion formula for the spherical transform,

$$
p_{t}=e^{\langle\rho, \rho\rangle t} \delta^{-1 / 2} q_{t}=C \int_{\mathfrak{a}^{*}} e^{-t\langle\lambda, \lambda\rangle}\left[\delta^{-1 / 2} \varphi_{\lambda}\right]|c(\lambda)|^{-2} d \lambda,
$$

where $C$ denotes the normalisation constant [4, Theorem 7.5]. So, by the mean value theorem, if $h>0$,

$$
\left\|p_{t+h}-p_{t}\right\| \leq C h \int_{a^{*}}\{1+\langle\lambda, \lambda\rangle\} e^{-t\langle\lambda, \lambda\rangle}|| \delta^{-1 / 2} \varphi_{\lambda} \||c(\lambda)|^{-2} d \lambda .
$$

However, the spherical functions $\varphi_{\lambda}$ are uniformly bounded for $\lambda$ in $\mathfrak{a}^{*}$, and $\delta^{-1 / 2} \in L^{2}\left(w_{s}\right)$ if $s$ is sufficiently large negative. It then follows that

$$
\left\|p_{t+h}-p_{t}\right\|_{L^{2}\left(w_{s}\right)} \leq C(t) h,
$$

where $C(t)$ is continuous in $t$.

Now pass to $u(t, \cdot)=p_{t} * f(\cdot)$. We have

$$
\|u(t+h, \cdot)-u(t, \cdot) \mid=\| \int_{S}\left\{p_{t+h}(\cdot y)-p_{t}(\cdot y)\right\} f\left(y^{-1}\right) d y \|,
$$

$S$ denoting the compact support of $f$. For each $y$ in $S$,

$$
\left\|p_{t+h}(\cdot y)-p_{t}(\cdot y)\right\|_{L^{2}\left(w_{s}\right)} \leq C\left\|p_{t+h}-p_{t}\right\|_{L^{2}\left(w_{s}\right)},
$$

where

$$
C=\sup _{z \in S}\left|\delta\left(z^{-1}\right) w_{-s}(z)\right|^{1 / 2} \text {. }
$$


It follows directly from (6) and (7) that the function $t \mapsto u(t, \cdot)$ satisfies the stated absolute continuity condition. The derivative condition is established along similar lines.

From now on we shall assume that the symmetric space $G / K$ satisfies one of the following conditions:

1. $G / K$ has rank one.

2. $G$ is a complex group.

3. $G$ is a normal real form.

Proposition 5.4. Let $\left\{p_{t}\right\}_{t>0}$ be the convolution semigroup of heat kernels (5). There exist a measure space $(B, \mathscr{B}, \mu)$, nonnegative measurable functions $\varphi$, $\psi_{1}, \ldots, \psi_{k}$ on $A N \times B$, and positive constants $a_{1}, \ldots, a_{k}$ such that

$$
p_{t}=\sum_{j=1}^{k} p_{t}^{(j)}
$$

where

$$
p_{t}^{(j)}(x)=t^{-a_{j}} \int_{B} e^{-\varphi(x, y) / t} \psi_{j}(x, y) d \mu(y)
$$

for all $x$ in $A N$ and $t$ in $\mathbf{R}^{+}$.

Proof. 1 . The case where $G / K$ has rank 1 . Then $G / K$ can be realised as the $n$-dimensional hyperbolic space $H_{n}(\mathbf{F})$ over $\mathbf{F}$, where $\mathbf{F}=\mathbf{R}, \mathbf{C}, \mathbf{H}$ or $\mathbf{O}$, i.e. the set of real numbers, of complex numbers, of quaternions or of Cayley octonions. We write $d=\operatorname{dim}_{\mathbf{R}} \mathbf{F}$ and $l=n d ; l$ is the dimension of $H_{n}(\mathbf{F})$ over R. Let $\alpha$ and $2 \alpha$ denote the positive roots with multiplities $m_{1}$ and $m_{2}$ respectively. Choose $H_{\alpha}$ in a such that $\alpha\left(H_{\alpha}\right)=1$. Write

$$
D_{x}=-\frac{1}{\sinh x} \frac{d}{d x} \text {. }
$$

The inversion formulae for the Abel transform on $H_{n}(\mathbf{F})$ [10, Hilfssatz 1] yield the following expressions for $q_{t}\left(\exp x H_{\alpha}\right)$, for any $x$ in $\mathbf{R}^{+}$:

(a) If $\mathbf{F}=\mathbf{R}$ and $l$ is odd (i.e. $d=1, l=n$ is odd)

$$
q_{t}\left(\exp \left(x H_{\alpha}\right)\right)=c t^{-1 / 2} e^{-\langle\rho, \rho\rangle t} D_{x}^{(l-1) / 2} e^{-x^{2} / 4 t} .
$$

(b) If $\mathbf{F}=\mathbf{R}$ and $l$ is even,

$$
q_{t}\left(\exp x H_{\alpha}\right)=c t^{-1 / 2} e^{-\langle\rho, \rho\rangle t} \int_{x}^{+\infty}(\cosh y-\cosh x)^{-1 / 2}\left(D_{y}^{l / 2} e^{-y^{2} / 4 t}\right) \sinh y d y \text {. }
$$

(c) If $\mathbf{F}=\mathbf{C}, \mathbf{H}$ or $O$, there exist positive constants $c_{1}, \ldots, c_{d / 2}$ such that

$$
\begin{array}{r}
q_{t}\left(\exp \left(x H_{\alpha}\right)\right)=c t^{-1 / 2} e^{-\langle\rho, \rho\rangle t} \sum_{j=1}^{d / 2} c_{j} \int_{x}^{+\infty}\left(\cosh ^{2} y-\cosh ^{2} x\right)^{-1 / 2}(\cosh y)^{j+1-d} \\
\times\left(D_{y}^{j+d(n-1) / 2} e^{-y^{2} / 4 t}\right) \sinh y d y .
\end{array}
$$


We define $\Phi_{r}$, for $r$ in $\mathbf{N}$, inductively by the formulae $\Phi_{1}(x)=x / \sinh x$ and

$$
\Phi_{r+1}(x)=D_{x} \Phi_{r}(x)
$$

for all $x$ in $\mathbf{R}$. Then a simple induction argument shows that, for every positive integer $m$,

$$
D_{x}^{m} e^{-x^{2} / 4 t}=\sum_{j=1}^{m} \psi_{j}(x) t^{-j} e^{-x^{2} / 4 t},
$$

where the functions $\psi_{j}$ are linear combinations, with positive coefficients, of products $\Phi_{r_{1}} \ldots \Phi_{r_{j}}$. Since

$$
\Phi_{r}(x)=\varphi_{0}^{(2 r+1)}\left(\exp \left(x H_{\alpha}\right)\right),
$$

where $\varphi_{0}^{(2 r+1)}$ is the spherical function on $H_{2 r+1}(\mathbf{R})$ corresponding to the eigenvalue $-\langle\rho, \rho\rangle$, the functions $\psi_{j}$ are positive. The desired expression for $p_{t}$ then follows from the respective formulae $(8)-(10)$ in the manner indicated below.

If $d=1$ and $n$ is odd (case (a)), simply take the one-point measure space for $(B, d \mu)$.

If $d=1$ and $n$ is even (case (b)), take $(B, d \mu)=\left(\mathbf{R}_{+}, d y\right)$ and perform the change of variables $u=\cosh y-\cosh x$.

Similarly, if $d>1$ (case (c)), take $(B, d \mu)=\left(\mathbf{R}_{+}, d y\right)$, and make the change of variables $u=\cosh ^{2} y-\cosh ^{2} x$.

2. The complex case. The heat kernel for $G / K$ is given by

$$
q_{t}(x)=C t^{-n / 2} e^{-\langle\rho, \rho\rangle t} \varphi_{0}(x) e^{-|x|^{2} / 4 t}
$$

for $x$ in $G / K$ [3]. Since the spherical function $\varphi_{0}$ is positive, the desired expression for $p_{t}$ follows from (14) if we take for $(B, d \mu)$ the one-point measure space.

3. Normal real forms. If the Lie algebra $\mathfrak{g}$ of $G$ is a normal real form of a complex Lie algebra $\tilde{\mathfrak{g}}$, the method of reduction to the complex case of Flensted-Jensen [3] (see also [1]) yields

$$
q_{t}(\exp H)=C \int_{K^{\mathrm{C}}} q_{t / 2}^{\mathrm{C}}(k \exp (H / 2)) d k
$$

for all $H$ in $\mathfrak{a}$, where $q_{t}^{\mathrm{C}}$ is the heat kernel for the complex case, and $K^{\mathrm{C}}$ is an analytic subgroup of $\widetilde{G}$, the analytic group whose Lie algebra is $\tilde{\mathfrak{g}}$. Let $\rho$ and $\widetilde{\rho}$ be half the sum of the positive roots of the pairs $(\mathfrak{g}, \mathfrak{a})$ and $(\tilde{\mathfrak{g}}, \tilde{\mathfrak{a}})$ respectively, and let $\langle\rangle,,\|\cdot\|,\langle,\rangle_{\sim}$, and $\|\cdot\|_{\sim}$ be the inner product and norm induced by the Killing forms on the respective Cartan subalgebras $\mathfrak{a}$ and $\tilde{\mathfrak{a}}$. Since $\mathfrak{g}$ is a normal real form of $\tilde{\mathfrak{g}}$, one has $\mathfrak{a}=\tilde{\mathfrak{a}}$. Moreover $\tilde{\rho}=2 \rho$ and $2\|H\|_{\sim}^{2}=\|H\|^{2}$ for all $H$ in $\mathfrak{a}$. Thus, by (14) and (15),

$$
q_{t}(\exp H)=C(t / 2)^{-n / 2} e^{-\langle\rho, \rho\rangle t} \int_{K^{\mathrm{C}}} \varphi_{0}(k \exp (H / 2)) e^{-|k \exp (H / 2)|^{2} / 4 t} d k .
$$


Once again, (5) yields the desired expression for $p_{t}$.

We come now to the maximal function theorem.

Theorem 5.5. For each of the three classes of groups $G$ described before the statement of Proposition 5.4, the maximal operator $\mathscr{M}$ for the heat kernels $\left\{p_{t}\right\}_{t>0}$ associated to the Laplacean $\Delta$ on $A N$ is of weak type $(1,1)$.

Proof. Recall the notation that $a_{t}=t^{-1} \int_{t}^{2 t} p_{s} d s$. By Proposition 5.4,

$$
a_{t}(x) \geq a_{t}^{(j)}=t^{-1} \int_{t}^{2 t} p_{s}^{(j)} d s \geq 2^{-a_{j}} p^{(j)}
$$

for $j=1, \ldots, k$. It follows from the Hopf-Dunford-Schwartz theorem that each of the maximal operators $\mathscr{M}_{j}$,

$$
\mathscr{M}_{j} f=\sup _{t>0}\left|p_{t}^{(j)} * f\right|,
$$

is of weak type $(1,1)$. So therefore is $\mathscr{M}$.

\section{REFERENCES}

1. J.-Ph. Anker and N. Lohoué, Multiplicateurs sur certains espaces symétriques, Amer. J. Math. 108 (1986), 1303-1354.

2. N. Dunford and J. T. Schwartz, Linear operators, Vol. I, Wiley, New York, 1957.

3. M. Flensted-Jensen, Spherical functions on a real semisimple Lie group. A method of reduction to the complex case. J. Funct. Anal. 30 (1978), 106-146.

4. S. Helgason, Groups and geometric analysis, Academic Press, London, 1984.

5. E. Hille and R. S. Phillips, Functional analysis on semi-groups, Amer. Math. Soc. Colloq. Publ., vol. 31, Amer. Math. Soc., Providence, R.I., 1957.

6. L. Hörmander, Hypoelliptic second-order differential equations, Acta Math. 119 (1967), 147171.

7. A. Hulanicki, Subalgebra of $L_{1}(G)$ associated with Laplacian on a Lie group, Colloq. Math. 31 (1974), 259-287.

8. J. W. Jenkins, A characterization of growth in locally compact groups, J. Funct. Anal. 12 (1973), 113-127.

9. S. Kusuoko and D. Stroock, Long time estimates for the heat kernel associated with a uniformly subelliptic symmetric second order operator, Ann. of Math. (2) 127 (1988), 165189.

10. N. Lohoué and Th. Rychener, Die Resolvente von $\Delta$ auf symmetrischen Raümen von nichtkompakten Typ, Comment. Math. Helv. 57 (1982), 445-468.

11. E. M. Stein, Topics in harmonic analysis related to the Littlewood-Paley Theory, Ann. of Math. Stud., no. 63, Princeton Univ. Press, Princeton, N.J., 1970.

12. N. Th. Varopoulos, Analysis on Lie groups, J. Funct. Anal. 76 (1988), 346-410.

School of Mathematics, University of New South Wales, Kensington N.S.W. 2033, Australia (Current address of Michael Cowling)

School of Mathematical Sciences, The Flinders University of South Australia, Bedford Park S.A. 5042, Australia (Current address of Garth Gaudry)

Dipartimento di Matematica, Università di Genova, 16132 Genova, Italy (Current address of Saverio Giulini and Giancarlo Mauceri) 